\title{
Intact parathyroid hormone and whole parathyroid hormone assay results disagree in hemodialysis patients under cinacalcet hydrochloride therapy
}

\author{
Ryo Koda $\cdot$ Junichiro James Kazama $\cdot$ Koji Matsuo Kazuko Kawamura • \\ Suguru Yamamoto $\cdot$ Minako Wakasugi $\cdot$ Tetsuro Takeda $\cdot$ Ichiei Narita
}

Received: 30 April 2013/Accepted: 17 October 2014/Published online: 11 November 2014

(C) The Author(s) 2014. This article is published with open access at Springerlink.com

\begin{abstract}
Background The parathyroid gland secretes 1-84 and 7-84 parathyroid hormone (PTH) fragments, and its regulation is dependent on stimulation of the extracellular calcium-sensing receptor. While the intact PTH system detects both PTH fragments, the whole PTH system detects the 1-84PTH but not the 7-84PTH. Cinacalcet hydrochloride $(\mathrm{CH})$ binds to calcium-sensing receptor as a calcimimetic. Here we investigated the role of $\mathrm{CH}$ treatment in the assessment of parathyroid gland function.

Methods Stable adult dialysis patients for whom $\mathrm{CH}$ therapy was planned were included. Patients for whom $\mathrm{CH}$ therapy was not planned were simultaneously included as the control group.

Results The $\mathrm{CH}$ group $(n=44)$ showed significantly higher circulating levels of $\mathrm{Ca}$, intact $\mathrm{PTH}$, and whole $\mathrm{PTH}$, before the $\mathrm{CH}$ treatment than the control group $(n=112)$. The $\mathrm{Ca}$, intact PTH, and whole PTH levels
\end{abstract}

R. Koda · J. J. Kazama · K. Matsuo · K. Kawamura ·

S. Yamamoto $\cdot$ M. Wakasugi $\cdot$ I. Narita

Department of Clinical Nephrology and Rheumatology, Niigata

University Graduate School of Medical and Dental Sciences,

Niigata, Japan

R. Koda $\cdot$ T. Takeda

Department of Nephrology, Dokkyo University Koshigaya

Medical Center, Koshigaya, Japan

\section{J. J. Kazama ( $₫)$}

Division of Blood Purification Therapy, Niigata University

Medical and Dental Hospital, 1-754 Asahimachi-Diri, Chuo-ku,

Niigata, Niigata 951-8510, Japan

e-mail: jjkaz@med.niigata-u.ac.jp

M. Wakasugi

Center of Interorgan Research, Niigata University Graduate

School of Medical and Dental Hospital, Niigata, Japan decreased along with the $\mathrm{CH}$ therapy, and the $\mathrm{Ca}$ levels became comparable in the 8 th week of treatment and thereafter. The $\mathrm{CH}$ group in the 8th week and thereafter showed significantly lower whole/intact PTH ratios than the control group, while the whole/intact PTH ratio was not significantly different between before and during the $\mathrm{CH}$ therapy. A multiple regression analysis revealed that the whole/intact PTH ratio was almost constant, but both the serum $\mathrm{Ca}$ level and a $\mathrm{CH}$ therapy could potentially modify the fixed number. When the whole PTH levels were estimated by intact PTH levels using the relationship between them in the control group, the levels were clearly overestimated in the $\mathrm{CH}$ group.

Conclusions Although the direct effect of $\mathrm{CH}$ on the whole/intact PTH ratio is masked by its hypocalcemic action, we could successfully demonstrate that the ratio in $\mathrm{CH}$ users is lower than that in the non-users with comparable levels of serum $\mathrm{Ca}$. Evaluating parathyroid function with intact PTH according to the clinical practice guidelines in patients being treated with $\mathrm{CH}$ may lead to significant overestimation and subsequent overtreatment.

Keywords Intact PTH - Whole PTH - Cinacalcet hydrochloride $\cdot$ Hemodialysis

\section{Introduction}

The secretion of parathyroid hormone (PTH) by parathyroid cells is controlled by extracellular calcium $(\mathrm{Ca})$ levels [1]. The sensor system that transmits the information of extracellular $\mathrm{Ca}$ levels into the nuclei of parathyroid cells involves the Ca-sensing receptor [2]. Cinacalcet hydrochloride $(\mathrm{CH})$ also binds to the $\mathrm{Ca}$-sensing receptor, and it transmits false information of elevated extracellular $\mathrm{Ca}$ 
levels into the cells [3]. As a result, $\mathrm{CH}$ suppresses the PTH secretion and production in parathyroid cells. $\mathrm{CH}$ is thus currently used for the treatment of secondary hyperparathyroidism in chronic kidney disease (CKD) patients with satisfactory clinical effects [4, 5].

In addition to $1-84 \mathrm{PTH}$, which actually induces hormonal action within the target cells, various PTH fragments of other lengths are also found in the systemic circulation. Among these fragments, 7-84PTH is directly secreted from parathyroid cells, and binds to PTH-I receptors with a binding affinity comparable to that of 1-84PTH [6]. The extracellular $\mathrm{Ca}$ level critically affects the switching of 1-84PTH/7-84PTH secretion in parathyroid cells. That is, 7-84PTH is predominantly secreted from parathyroid cells under a hypercalcemic condition, whereas 1-84PTH secretion is promoted in these cells under a hypocalcemic condition [7, 8]. Since total PTH levels are also increased under a hypocalcemic condition, the change in the serum 1-84PTH level is greatly enhanced by the extracellular $\mathrm{Ca}$ level. Because the extracellular $\mathrm{Ca}$ levels are sensed through the Ca-sensing receptor, it has been hypothesized that in patients being treated with $\mathrm{CH}$, the secretion of $1-84 \mathrm{PTH}$ is suppressed, while 7-84PTH secretion is promoted.

The so-called intact PTH assay detects both 1-84PTH and 7-84PTH fragments. In contrast, the 3rd generation PTH assay, also known as the whole PTH assay, does not detect 7-84PTH fragments because one of the epitopes for the sandwich immunoassay is located at the very N-terminal of the PTH molecule [9]. Strictly speaking, the whole PTH assay does detect some fragments other than true 1-84 PTH [10]. However, the majority of investigators would agree that the whole PTH assay detects functioning PTH molecules more specifically than the intact PTH assay does.

The levels of intact PTH and whole PTH do show a tight correlation [11]. However, the whole/intact PTH ratio, which is practically indicating the ratio of 1-84PTH and the sum of $1-84 \mathrm{PTH}+7-84 \mathrm{PTH}$, varies widely among individuals, and some clinical investigators insist that the ratio is an indicator of bone turnover [12]. In addition, vitamin D receptor activator treatment has been shown to slightly decrease the whole/intact PTH ratio in dialysis patients with secondary hyperparathyroidism, presumably through its calcemic action [13]. Since, $\mathrm{CH}$ therapy directly suppresses 1-84PTH secretion while promoting 7-84PTH secretion, in theory, its effect on the whole/intact $\mathrm{PTH}$ ratio change might be much greater. If $\mathrm{CH}$ therapy has a major effect on the whole/intact $\mathrm{PTH}$ ratio, then it would no longer be possible to estimate 1-84PTH levels from intact PTH levels.

To address these issues, we performed a prospective clinical study. The aim of this study was to validate the efficacy of PTH assays for evaluating parathyroid function in dialysis patients under $\mathrm{CH}$ therapy.

\section{Patients and methods}

\section{Patients}

Adult CKD patients who had been under stable maintenance hemodialysis therapy for more than 1 year at Niigata University Medical and Dental Hospital or one of its related facilities were enrolled. All subjects were dialysis patients for whom $\mathrm{CH}$ therapy was considered due to prolonged hyperactivation of parathyroid function despite of other medical therapy in accordance with the Clinical Practice Guidelines for CKD-MBD issued by the Japanese Society for Dialysis Therapy (JSDT guidelines) [14]. The exclusion criteria were as follows: (1) hypocalcemia defined by serum Ca levels $<9.0 \mathrm{mg} / \mathrm{dL}$; (2) the presence of any contraindications for $\mathrm{CH}$ therapy; (3) past history of kidney transplantation or parathyroid intervention therapies; and (4) complications of malignant tumor, severe cardiac failure, chronic inflammatory disease, or any other diseases that could disturb stable hemodialysis therapy. All the enrolled patients provided written informed consent to participate in the study. With respect to the registration of $\mathrm{CH}$ patients, the simultaneous registration of one or more control patients with mild secondary hyperparathyroidism, who had not received and would not need $\mathrm{CH}$ therapy, was recommended. However, the registration of control patients was not compulsory if no applicable patients were available.

\section{Methods}

All the patients' blood samples were collected at 9:00 a.m. after a 12-h period of fasting. The initial dosage of $\mathrm{CH}$ was $25 \mathrm{mg} /$ day. In accordance with the JSDT guidelines, the dosage of $\mathrm{CH}$ was thereafter freely changed in order to maintain the intact PTH levels in the patients in the $\mathrm{CH}$ group. However, the minimal dosage was set at $12.5 \mathrm{mg} /$ day, and those patients who required a decrease in the dosage of $\mathrm{CH}$ to $<12.5 \mathrm{mg}$ were excluded from the analyses. No limitation was set for using vitamin D receptor activators, phosphate binders, antihypertensive agents, or erythropoietin receptor stimulating agents.

Blood samples were obtained from the $\mathrm{CH}$ patients before the $\mathrm{CH}$ therapy was initiated $(0 \mathrm{~W})$, and at $4,8,12$, 16,24 , and 48 weeks after the initiation of the $\mathrm{CH}$ therapy. Data obtained from the $\mathrm{CH}$ group were subjected to the analyses only at the time points when the serum calcium concentration was comparable to that in the control group (Fig. 1). 


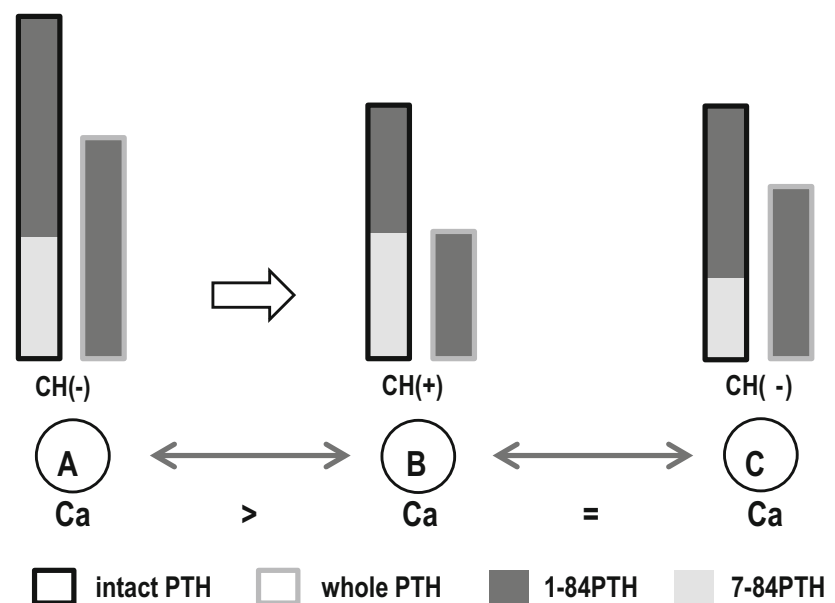

Fig. 1 Generally, the serum Ca concentration decreased along with the time course of $\mathrm{CH}$ therapy. Since the serum $\mathrm{Ca}$ level is another important determinant of the whole/intact PTH ratio, the pure effect of $\mathrm{CH}$ on the whole/intact PTH ratio cannot be evaluated by comparing the data obtained from the same patients between before $(A)$ and during/after $(B) \mathrm{CH}$ therapy. Therefore, we established a control group of patients $(C)$ whose parathyroid functions were stable even without using $\mathrm{CH}$ in this protocol. When the serum $\mathrm{Ca}$ levels became comparable between $B$ and $C$, then the only difference in the major determinant of the whole/intact PTH ratio was the use of $\mathrm{CH}$, and thus we could evaluate the pure effect of $\mathrm{CH}$

Intact PTH was detected by an electrochemiluminescence immunocomparable assay (Roche Diagnostics, Tokyo), and whole PTH was detected by an immunoradiometric assay (DS Pharma Biomedical Co., Osaka). For the control group, the blood sample collection was performed only once.

The data are expressed as the mean (SD). Comparisons between the control group and the $\mathrm{CH}$ group were performed with an unpaired $t$ test. Comparisons within the $\mathrm{CH}$ groups were performed with a paired $t$ test. A $p$ value $<.05$ was considered significant.

In order to simulate the clinical influence of the alteration of the whole/intact PTH ratio by $\mathrm{CH}$ therapy, we performed an analysis as follows. Parathyroid function in the patients was diagnosed by their intact PTH levels according to the JSDT guidelines, namely, hypoparathyroid condition $=$ intact $\quad$ PTH $<60 \mathrm{pg} / \mathrm{Ll}$, normoparathyroid condition $=$ intact PTH levels between 60 and $240 \mathrm{pg} / \mathrm{mL}$, and hyperparathyroid condition $=$ intact $\mathrm{PTH}$ levels more than $240 \mathrm{pg} / \mathrm{mL}$. Similarly, the patients were diagnosed by their whole PTH levels according to the JSDT guidelines, namely, hypoparathyroid condition $=$ whole PTH levels $<35 \mathrm{pg} / \mathrm{mL}$, normoparathyroid condition $=$ whole $\mathrm{PTH}$ levels between 35 and $150 \mathrm{pg} / \mathrm{mL}$, and hyperparathyroid condition $=$ whole PTH levels more than $150 \mathrm{pg} / \mathrm{mL}$. When these diagnoses were in agreement with each other, the diagnosis was regarded as successful. Those cases with hypoparathyroid conditions measured by the intact PTH

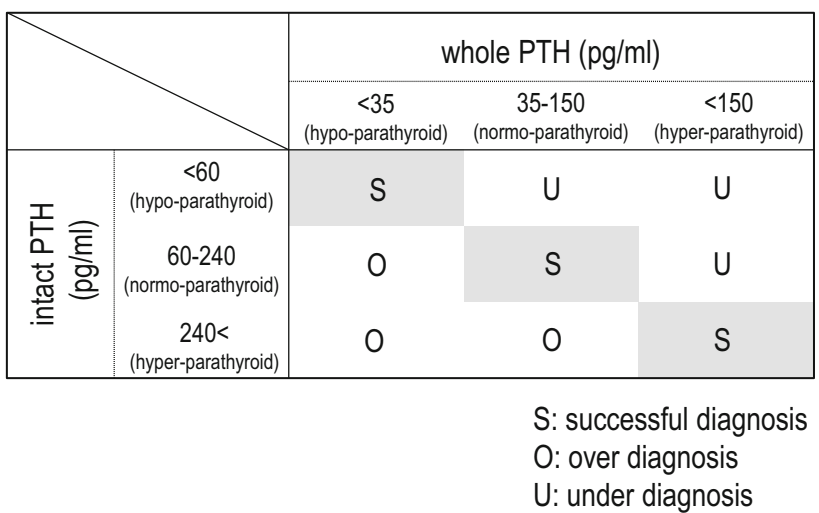

Fig. 2 Analyzing principle used to assess the effect of the whole/ intact PTH change in clinical practice. The JSDT guidelines set two standard PTH levels: intact PTH levels between 60 and $240 \mathrm{pg} / \mathrm{mL}$ and whole PTH levels between 35 and $150 \mathrm{pg} / \mathrm{mL}$. If the relationship between these two assay results is always constant, the diagnoses made by these two standard levels would agree, and therefore the patients would be classified into successful diagnosis $(S)$ in the majority of cases. However, if $\mathrm{CH}$ therapy modifies the relationship between these assay results, the numbers of cases classified into under-diagnosis $(U)$ or over-diagnosis $(O)$ may be increased, which becomes a potential cause of false diagnosis at bedside

assay and normo- or hyperparathyroid conditions measured by the whole PTH assay, as well as those cases with hypoor normoparathyroid conditions as shown by the intact PTH assay and hyperparathyroid condition shown by the whole PTH assay, were considered to have an underestimation of whole PTH by the conversion formula with intact PTH. The patients with normo- or hyperparathyroid conditions by intact PTH and hypoparathyroid condition by whole PTH, as well as those with hyperparathyroid condition by intact PTH and hypo- or normoparathyroid conditions by whole PTH, were considered to have an overestimation of whole PTH by the conversion formula with intact PTH (Fig. 2). The above analyses were performed in both the control and $\mathrm{CH}$ groups, and the ratios of successful diagnosis, under-diagnosis, and over-diagnosis were compared between them.

This study was designed in accordance with the Helsinki Declaration. All participants in both the control and $\mathrm{CH}$ groups received an adequate explanation of the study design, and written consent was obtained before the initiation of the study. This study protocol was approved by the Ethics Committee of Niigata University (\#1028). The data presented in this manuscript were managed only by the authors or the cooperating personnel listed in the Acknowledgment section.

\section{Results}

Sixty-seven patients were originally registered for the study as the $\mathrm{CH}$ group. Of these $67, \mathrm{CH}$ treatment was 
discontinued due to over-suppression of parathyroid function in 11 patients, nausea in 4, transfer to another dialysis clinic in 4, and for other reasons in 2 patients. Parathyroid intervention therapy was indicated in 1 patient. Data were inappropriately collected from 1 patient. After excluding the above 23 patients, 44 patients were included in the final analysis. For the control group, 113 subjects were enrolled, and the data were appropriately collected from 112 patients who were included in the analysis. Table 1 shows the clinical back grounds of the $\mathrm{CH}$ group and control group patients. Before the $\mathrm{CH}$ treatment was initiated, the serum $\mathrm{Ca}$ levels were significantly higher in the $\mathrm{CH}$ group than those in the

Table 1 The clinical background of the patients in the $\mathrm{CH}$ group and control groups

\begin{tabular}{llll}
\hline & Control $(N=112)$ & $\mathrm{CH}(N=44)$ & $p$ \\
\hline Sex & M70:F42 & M28:F16 & .896 \\
Age & $64.2(12.7)$ & $63.8(12.4)$ & .835 \\
Dialysis vintage (Y) & $12.0(5.5)$ & $12.4(7.9)$ & .697 \\
Diabetes mellitus (\%) & 21.4 & 20.5 & .894 \\
\hline
\end{tabular}

control group. The $\mathrm{CH}$ group also showed higher serum levels of intact PTH and whole PTH, and lower whole/ intact PTH ratios.

In the $\mathrm{CH}$ group, the dosages of active vitamin $\mathrm{D}$ agents, phosphate binders, and antihypertensive agents were generally unchanged in the $\mathrm{CH}$ group, but the dosage of $\mathrm{CH}$ was slightly increased along with the time course (Table 2). The serum levels of $\mathrm{Ca}, \mathrm{Pi}$, intact PTH, and whole PTH decreased along with the $\mathrm{CH}$ therapy. Although the $\mathrm{CH}$ group showed higher serum $\mathrm{Ca}$ levels before the initiation of $\mathrm{CH}$ therapy, the levels became comparable to the control group at the 8th week and thereafter (Fig. 3). Therefore, we compared the data obtained from the control group and the $\mathrm{CH}$ group at weeks $8,12,16,24$, and 48 .

Figure 4 demonstrates the relationship between intact PTH and whole PTH in the control and $\mathrm{CH}$ groups at weeks 8, 12, 16, 24, and 48. Although these parameters were tightly correlated within both groups at all-time points, the regression lines were significantly less steep in the $\mathrm{CH}$ group. The $\mathrm{CH}$ group showed significantly lower whole/intact PTH ratios compared with the control group. However, the whole/intact PTH ratio was not significantly

Table 2 The medication profile of the $\mathrm{CH}$ group patients and the control group

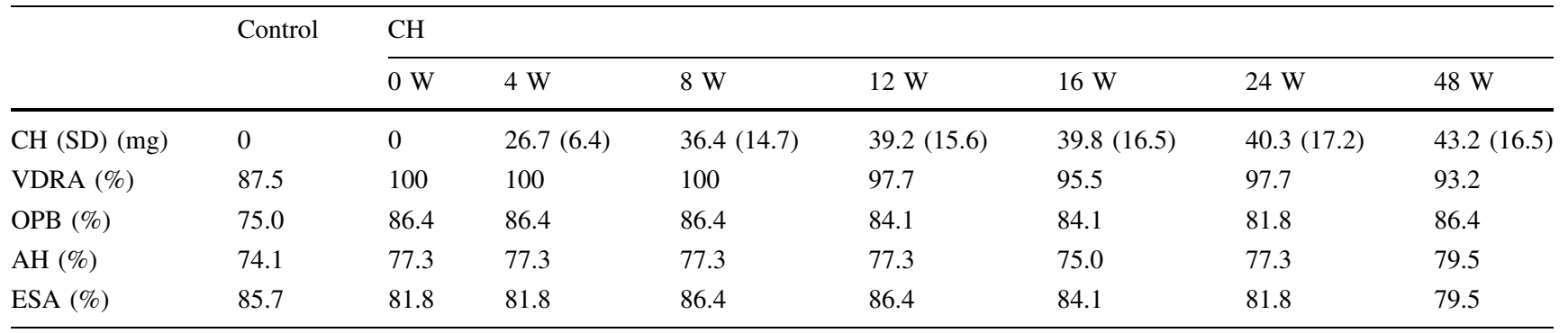

$C H$ cinacalcet hydrochloride, $V D R A$ vitamin D receptor activator, $A H$ anti-hypertensive agents, $O P B$ oral phosphate binders, $E S A$ erythropoiesis stimulating agents

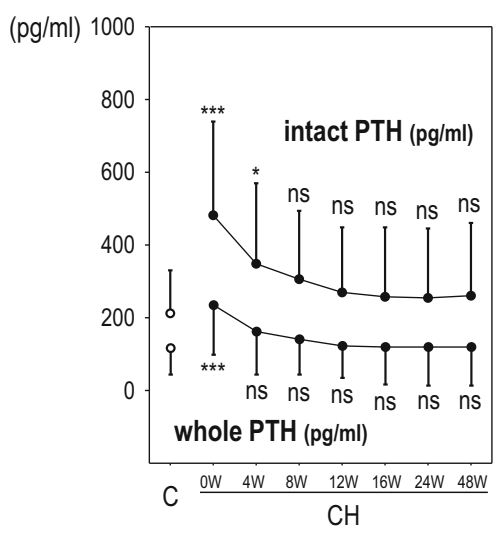

Fig. 3 The changes in biochemical data during $\mathrm{CH}$ therapy. The serum levels of $\mathrm{Ca}, \mathrm{Pi}$, intact $\mathrm{PTH}$, and whole $\mathrm{PTH}$ decreased along with the $\mathrm{CH}$ therapy. Although the $\mathrm{CH}$ group showed higher serum $\mathrm{Ca}$ levels before the initiation of the $\mathrm{CH}$ therapy, the levels became

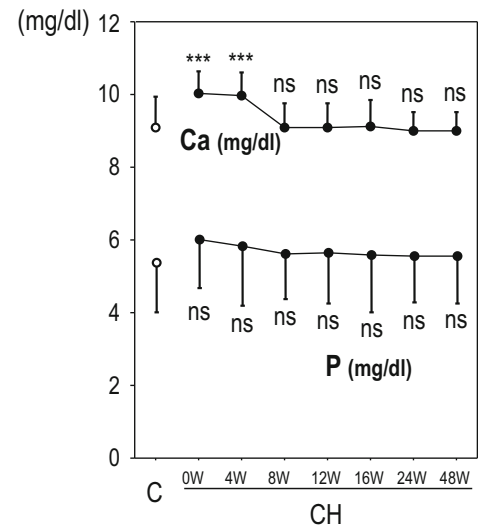

comparable with the control group at the 8 th week and thereafter. $C$ the control group, $\mathrm{CH}$ the cinacalcet hydrochloride group. ${ }^{*} p<.05$, $* * * p<.001$ versus $\mathrm{C}$ 

between intact PTH and whole PTH in the control and $\mathrm{CH}$ groups at weeks $8,12,16,24$, and 48. These values all showed tight correlations; however, the regression lines were steeper in the $\mathrm{CH}$ groups. ${ }^{*} p<.05$ versus the steepness in the control group
Fig. 4 The relationship
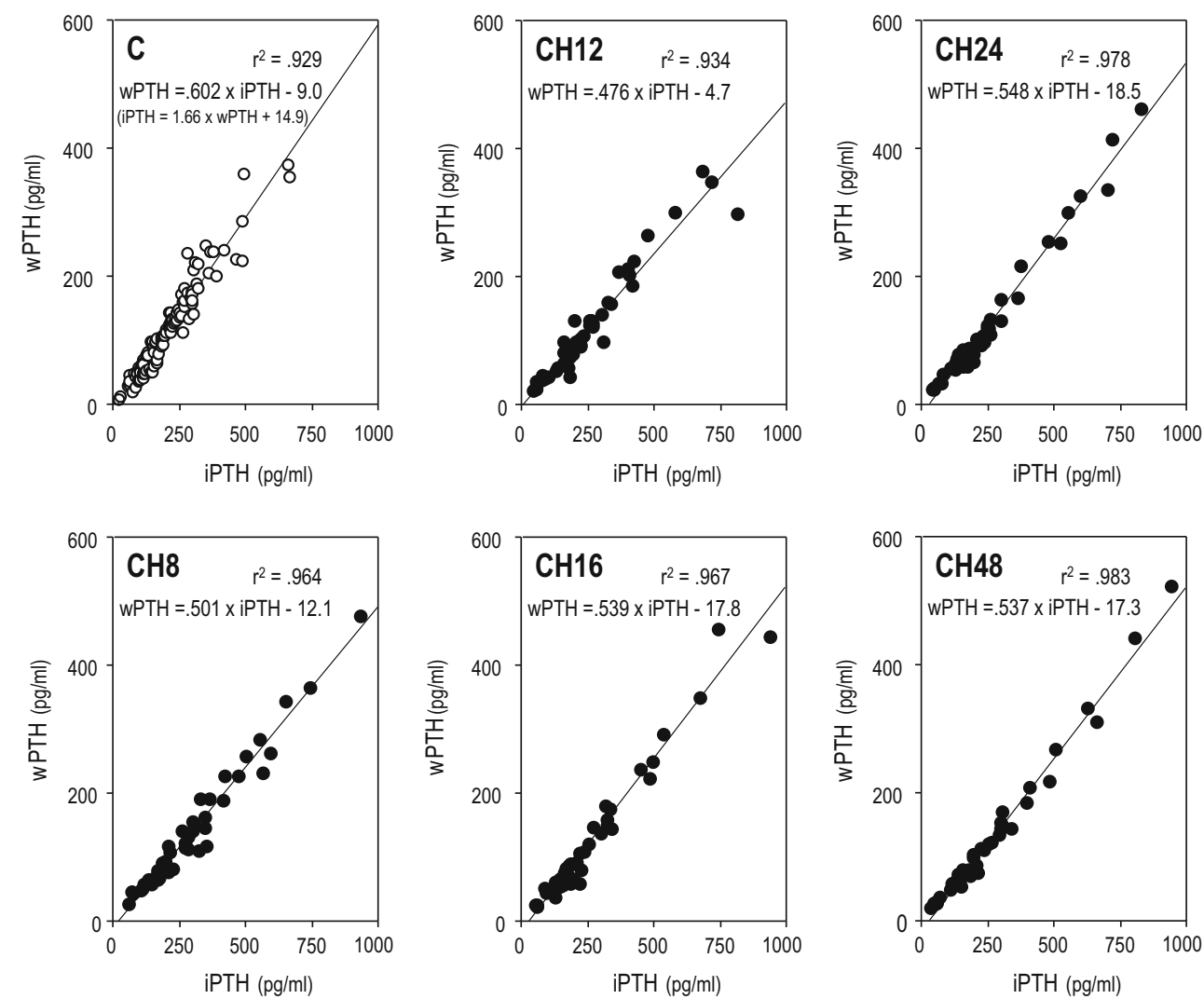

different between those before and during the $\mathrm{CH}$ therapy (Fig. 5). The results of the multiple regression analyses are demonstrated in Table 3. When we analyzed the control patients together with the $\mathrm{CH}$ group patients before the $\mathrm{CH}$ treatment, we identified the circulating levels of intact PTH and $\mathrm{Ca}$ as critical factors determining the circulating whole PTH levels. A comparable result was obtained when the control patients together with the $\mathrm{CH}$ group patients at the 48th week were analyzed with the same model. However, when $\mathrm{CH}$ treatment, the interaction term between intact PTH and $\mathrm{CH}$ (IT iPTH* $\mathrm{CH}$ ), the interaction term between intact PTH and $\mathrm{Ca}\left(\mathrm{IT} \mathrm{iPTH}^{*} \mathrm{Ca}\right)$, and the interaction term between $\mathrm{CH}$ and $\mathrm{Ca}$ (IT $\mathrm{CH}^{*} \mathrm{Ca}$ ) were added to the model as independent variables, only intact $\mathrm{PTH}$, IT iPTH* $\mathrm{CH}$, and IT $\mathrm{iPTH}^{*} \mathrm{Ca}$ were indicated as significant factors determining the circulating whole PTH levels.

In the control group, the diagnosis of parathyroid condition obtained based on the intact PTH assay successfully accorded with that obtained based on the whole PTH assay in more than $90 \%$ of the cases. In contrast, the rate of successful diagnosis declined to $<80 \%$ among those under $\mathrm{CH}$ therapy. All of the unsuccessful cases were overestimations of whole PTH by the conversion formula with intact PTH (Table 4).

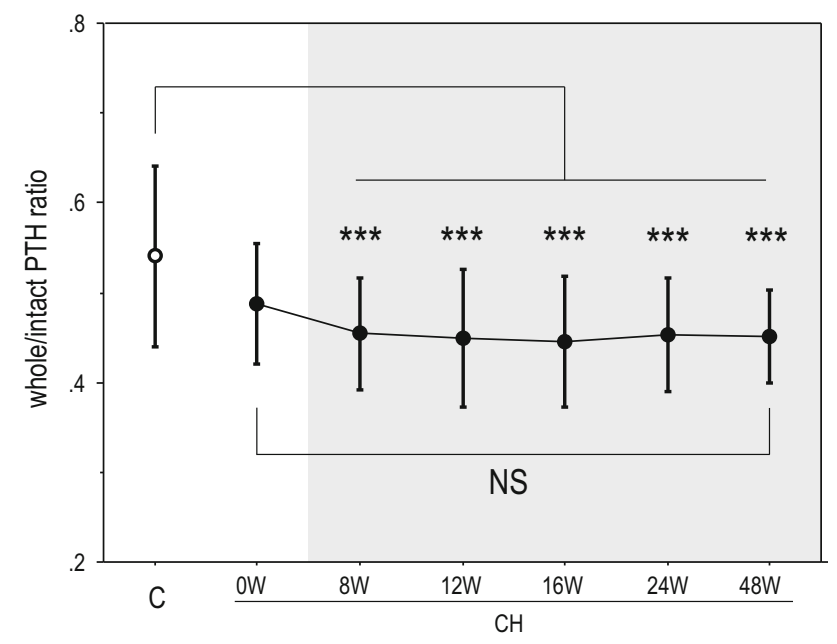

Fig. 5 The comparison of the whole/intact PTH ratios. The $\mathrm{CH}$ at 8 , $12,16,24$, and 48 weeks showed significantly lower whole/intact PTH ratios than the control group. Although the serum Ca levels were different between the control and the $\mathrm{CH}$ groups at $0 \mathrm{~W}$, the difference in the whole/intact PTH ratios did not reach significant level. The serum Ca levels were comparable between the control and $\mathrm{CH}$ groups at the 8th week and thereafter. Therefore, the difference in the whole/intact PTH ratios between these groups would be attributable solely to the $\mathrm{CH}$ therapy. On the other hand, the whole/intact PTH ratios were not significantly different between those before and during the $\mathrm{CH}$ therapy. ${ }^{* *} p<.01, * * * p<.001$ versus $\mathrm{C}$ 
Table 3 Results of the multiple regression analyses

\begin{tabular}{|c|c|c|c|}
\hline & $T$ & $p$ & $95 \% \mathrm{CI}$ \\
\hline \multicolumn{4}{|l|}{ (A) } \\
\hline iPTH & 51.074 & $<.0001$ & .500 to .540 \\
\hline $\mathrm{Ca}$ & -4.374 & $<.0001$ & -14.954 to -5.647 \\
\hline Age & 1.050 & .296 & -.155 to 506 \\
\hline Sex & .858 & .392 & -4.696 to 11.911 \\
\hline Vintage & .818 & .415 & .336 to 1.813 \\
\hline $\mathrm{DM}$ & .635 & .526 & -6.815 to 13.270 \\
\hline $\mathrm{P}$ & -.455 & .650 & -3.666 to 2.294 \\
\hline \multicolumn{4}{|l|}{ (B) } \\
\hline iPTH & 49.976 & $<.0001$ & .537 to .585 \\
\hline $\mathrm{Ca}$ & -2.068 & .040 & -9.132 to -.207 \\
\hline $\mathrm{P}$ & -1.464 & .145 & -4.548 to .677 \\
\hline Age & .842 & .401 & -.161 to 400 \\
\hline Vintage & -.455 & .650 & -.707 to 442 \\
\hline $\mathrm{DM}$ & .035 & .972 & -8.411 to 8.715 \\
\hline Sex & .002 & .998 & -7.080 to 7.094 \\
\hline \multicolumn{4}{|l|}{ (C) } \\
\hline iPTH & 8.862 & $<.0001$ & .857 to 1.349 \\
\hline IT iPTH*CH & -4.194 & $<.0001$ & -.122 to -.044 \\
\hline IT iPTH*Ca & -3.991 & .0001 & -.078 to -.026 \\
\hline $\mathrm{Ca}$ & 1.849 & .067 & -.448 to 13.426 \\
\hline $\mathrm{P}$ & -1.775 & .078 & -4.022 to .216 \\
\hline Age & 1.314 & .191 & -.075 to 372 \\
\hline $\mathrm{DM}$ & .618 & .537 & -4.748 to 9.071 \\
\hline $\mathrm{CH}$ & -.249 & .803 & -108.794 to 84.415 \\
\hline IT $\mathrm{CH}^{*} \mathrm{Ca}$ & .167 & .867 & -9.909 to 11.743 \\
\hline Sex & -.115 & .909 & -5.961 to 5.306 \\
\hline Vintage & .100 & .920 & -.037 to 041 \\
\hline
\end{tabular}

$A$ We analyzed the control patients together with the $\mathrm{CH}$ group patients before the $\mathrm{CH}$ treatment. Their age, sex, dialysis vintage, diabetes mellitus, circulating levels of intact $\mathrm{PTH}, \mathrm{Ca}$, and $\mathrm{Pi}$ were applied as the independent variables, and the whole PTH levels were set as the bound variable. As a result, intact PTH and $\mathrm{Ca}$ were identified as two significant factors determining the whole PTH levels

$B$ The control patients together with the $\mathrm{CH}$ group patients at the 48th week were analyzed with the same model as that shown in A. Again, intact PTH and Ca were identified as two significant factors

$C$ The $\mathrm{CH}$ treatment, the interaction term between intact PTH and $\mathrm{CH}$ (IT iPTH* $\mathrm{CH}$ ), that between intact PTH and $\mathrm{Ca}(\mathrm{IT}$ iPTH* $\mathrm{Ca}$ ), and that between $\mathrm{CH}$ and $\mathrm{Ca}$ (IT $\mathrm{CH}^{*} \mathrm{Ca}$ ) were added as independent variables to the model shown in B. Intact PTH, IT iPTH* ${ }^{*} \mathrm{H}$, and IT iPTH*Ca were confirmed to be significant factors determining the whole PTH levels

$D M$ comorbid condition of diabetes mellitus, vintage dialysis vintage

\section{Discussion}

It is the extracellular $\mathrm{Ca}$ level that plays the central role in switching 1-84PTH/7-84PTH secretions in parathyroid cells $[1,7,8]$, and $\mathrm{CH}$ may modify the mechanism through Ca-sensing receptor activation. Therefore, the switching of
Table 4 The effect of the $\mathrm{CH}$ therapy on the accordance of diagnoses made based on the intact PTH and whole PTH assay results

\begin{tabular}{llll}
\hline & $\begin{array}{l}\text { Successful } \\
\text { diagnosis }(\%)\end{array}$ & Over diagnosis $(\%)$ & $\begin{array}{l}\text { Under } \\
\text { diagnosis }\end{array}$ \\
\hline Cont & 91.1 & 8.9 & 0 \\
CH & & & \\
$8 \mathrm{~W}$ & 77.3 & $22.7^{* *}$ & 0 \\
$16 \mathrm{~W}$ & 79.5 & $20.5^{*}$ & 0 \\
$24 \mathrm{~W}$ & 75.0 & $25.0^{* *}$ & 0 \\
$48 \mathrm{~W}$ & 77.3 & $22.7^{* *}$ & 0 \\
\hline
\end{tabular}

In the control group (C), the diagnosis of parathyroid condition by the intact PTH successfully accorded with that by whole PTH in more than $90 \%$ of the cases. However, the rate of successful diagnosis fell to $<80 \%$ among those under the $\mathrm{CH}$ therapy $(\mathrm{CH})$. All of the unsuccessful cases were overestimation of whole PTH by the conversion formula with intact PTH

$* p<.05$, ** $p<.01$ versus $\mathrm{C}$

1-84PTH/7-84PTH secretions in patients under $\mathrm{CH}$ therapy is controlled by two major factors: extracellular $\mathrm{Ca}$ and $\mathrm{CH}$. The principal function of $\mathrm{CH}$ in parathyroid function is, strictly speaking, not the suppression of parathyroid cell function, but the alteration of the sigmoid curve between extracellular Ca stimuli and PTH secretion [15]. By considering this fact, we can explain why the previous research failed to demonstrate a change in the whole/intact PTH ratio by $\mathrm{CH}$ therapy [16]. $\mathrm{CH}$ suppressed $1-84 \mathrm{PTH}$ secretion and promoted 7-84PTH secretion. However, $\mathrm{CH}$ therapy also displayed hypocalcemic action [17], and the decrease in extracellular $\mathrm{Ca}$ levels promoted 1-84PTH secretion. Thus, Ca counteracted the 1-84PTH secretion against $\mathrm{CH}$, and therefore, the effect of $\mathrm{CH}$ on the whole/ intact PTH ratio became difficult to assess by comparing data before and during $\mathrm{CH}$ therapy in the same patients. In fact, just as in the previous study, the whole/intact PTH ratios before the $\mathrm{CH}$ therapy were comparable to those during the therapy in the present study as well (Fig. 3).

Thus, we established a group of control patients who did not need to be treated with $\mathrm{CH}$, and we compared the data obtained from the $\mathrm{CH}$ group with that from the control group, instead of comparing the data obtained from the same patients before and during $\mathrm{CH}$ therapy. However, we have to admit that the selection criteria for the control patients were not clear enough for our scientific analyses, ant this is the largest limitation of this study.

To establish the control group, we selected patients with mild secondary hyperparathyroidism who did not need $\mathrm{CH}$ therapy. Since CH therapy decreases serum Ca levels, it is generally not chosen for relatively hypocalcemic patients. In the present study, therefore, relatively hypercalcemic patients were enrolled as the $\mathrm{CH}$ group. The difference in the whole/intact PTH ratios between the control group and 
$\mathrm{CH}$ group at $0 \mathrm{~W}$ could be explained by the difference in their serum Ca levels (Fig. 2).

Since the difference in serum $\mathrm{Ca}$ levels between the treatment and control groups disappeared at week 8 , and thereafter, it became possible to compare the effects of $\mathrm{CH}$ at these time points. As a result, $\mathrm{CH}$ therapy was found to decrease the whole/intact PTH ratio (Fig. 2).

The intact PTH levels and whole PTH levels showed a tight correlation in the control group, as we expected, and the regression formula was as follows: intact PTH $=1.711 \times$ whole PTH (Fig. 3). This formula is similar to the conversion formula that appeared in the original version of the JSDT guidelines: intact $\mathrm{PTH}=1.7 \times$ whole PTH [17]. The intact PTH levels and whole PTH levels also showed tight linear correlations in the $\mathrm{CH}$ group, but the steepness of the regression line increased according to the changes in the whole/intact PTH ratio. The results presented in Table $3 \mathrm{C}$ are expressed as follows:

$$
\begin{aligned}
\text { whole } \mathrm{PTH}= & \mathrm{A} 1 \times(\text { intact } \mathrm{PTH})+\mathrm{A} 2 \times(\mathrm{IT} \text { iPTH } * \mathrm{CH}) \\
& +\mathrm{A} 3 \times(\mathrm{IT} \mathrm{iPTH} * \mathrm{Ca}) \\
= & (\mathrm{A} 1+\mathrm{A} 2 \times \mathrm{CH}+\mathrm{A} 3 \times \mathrm{Ca}) \times \text { int } \mathrm{PTH},
\end{aligned}
$$

where $\mathrm{A} 1, \mathrm{~A} 2$, and $\mathrm{A} 3$ indicate fixed numbers, respectively. This formula is converted as: the whole $\mathrm{PTH} / \mathrm{intact}$ $\mathrm{PTH}$ ratio $=\mathrm{A} 1+\mathrm{A} 2 \times \mathrm{CH}+\mathrm{A} 3 \times \mathrm{Ca}$. Therefore, $\mathrm{CH}$ was indicated as a potential bias besides $\mathrm{Ca}$ in the estimation of the whole PTH levels from the intact PTH levels.

Table 4 demonstrates the potential effect of the above changes in clinical practice. The diagnosis by intact PTH accorded with that by whole PTH in over $90 \%$ of the patients in the control group. This result supported the conventional concept that parathyroid function could be assessed by either the intact PTH or the whole PTH. The JSDT guidelines set the standard parathyroid function range of both intact PTH and whole PTH levels based on the premise that these two diagnoses always agree. However, the evidence adopted in these clinical practice guidelines was all obtained before $\mathrm{CH}$ became available in clinical practice $[14,18]$. Therefore, the relationship between intact PTH and whole PTH described by the JSDT guidelines is that between these levels under a condition in which $\mathrm{CH}$ is not administered, namely, the relationship found in the control group in the present study.

On the other hand, the agreement of these two diagnoses was decreased to $<80 \%$ in the $\mathrm{CH}$ group. All the disagreement cases overestimated the whole PTH levels by intact PTH levels (Table 4). This result was caused by the alteration of the relationship between intact PTH and whole PTH brought about by the $\mathrm{CH}$ therapy; in other words, the standard PTH levels expressed with intact PTH and those expressed with whole PTH indicate differing parathyroid functions under this condition. Since these patients were being treated with $\mathrm{CH}$ because of hyperparathyroidism, the impact of the potential unreliability of PTH assessment is great. If intact PTH levels indicate the true parathyroid function, the parathyroid suppression therapy would become under-treatment in patients whose parathyroid functions are monitored by whole PTH levels. If whole PTH indicates the true parathyroid function, $\mathrm{CH}$ therapy may tend to become over-treatment in those patients for whom intact PTH is used as a monitoring tool.

At present, it is uncertain whether intact PTH or whole PTH more accurately indicates true parathyroid function. However, the conventional concept that we can use either intact PTH or whole PTH for parathyroid monitoring cannot be applied under these conditions. Therefore, the time has come to choose the better PTH assay for standard use. In theory, the whole PTH assay seems to show stable results even under these conditions. Moreover, the intact PTH assays show greater interassay heterogeneities than the whole PTH assays $[19,20]$. However, further clinical investigations will be needed to select the better choice for bedside use.

In conclusion, the whole/intact PTH ratio in the dialysis patients under $\mathrm{CH}$ therapy was significantly lower than that in the control dialysis patients with comparable serum $\mathrm{Ca}$ levels. For patients under $\mathrm{CH}$ therapy, we must consider the most appropriate assay to assess parathyroid function, and we should not blindly apply the present clinical practice guidelines.

Acknowledgments The authors are grateful to Dr. Ikuo Aoike, Dr. Ryuji Aoyagi, Dr. Hitoshi Igarashi, Dr. Yumi Ito, Dr. Yoichi Iwafuchi, Dr. Isei Ei, Dr. Kiyoko Hosaka, Dr. Michihiro Hosojima, Dr. Kaoru Oya, Dr. Hideo Okajima, Dr. Asa Ogawa, Dr. Yuko Oyama, Dr. Ryohei Kaseda, Dr. Shigemi Kameda, Dr. Yutaka Koda, Dr. Hideyuki Kobayashi, Dr. Daisuke Kondo, Dr. Yuichi Sakamaki, Dr. Nobuyuki Sakurai, Dr. Masashi Suzuki, Dr. Hisaki Shimada, and Dr. Takuma Takata for cooperation and helpful discussion in the course of this study.

Conflict of interest JJK, TT, and IN have received financial support for clinical research from Kyowahakko-Kirin Co Ltd., which is the supplier of cinacalcet hydrochloride.

Open Access This article is distributed under the terms of the Creative Commons Attribution License which permits any use, distribution, and reproduction in any medium, provided the original author(s) and the source are credited.

\section{References}

1. Kumar R, Thompson JR. The regulation of parathyroid hormone secretion and synthesis. J Am Soc Nephrol. 2011;22:216-24.

2. Brown EM, MacLeod RJ. Extracellular calcium sensing and extracellular calcium signaling. Physiol Rev. 2001;81:239-97.

3. Nagano N, Nemeth EF. Functional proteins involved in regulation of intracellular $\mathrm{Ca}(2+)$ for drug development: the 
extracellular calcium receptor and an innovative medical approach to control secondary hyperparathyroidism by calcimimetics. J Pharmacol Sci. 2005;97:355-60.

4. Block GA, Martin KJ, de Francisco AL, Turner SA, Avram MM, Suranyi MG, et al. Cinacalcet for secondary hyperparathyroidism in patients receiving hemodialysis. $\mathrm{N}$ Engl $\mathrm{J}$ Med. 2004;350:1516-25.

5. Fukagawa M, Yumita S, Akizawa T, Uchida E, Tsukamoto Y, Iwasaki M, et al. Cinacalcet (KRN1493) effectively decreases the serum intact PTH level with favorable control of the serum phosphorus and calcium levels in Japanese dialysis patients. Nephrol Dial Transplant. 2008;23:328-35.

6. D'Amour P. Circulating PTH molecular forms: what we know and what we don't. Kidney Int. 2006;S29-S33.

7. Murray TM, Rao LG, Divieti P, Bringhurst FR. Parathyroid hormone secretion and action: evidence for discrete receptors for the carboxyl-terminal region and related biological actions of carboxyl-terminal ligands. Endocr Rev. 2005;26:78-113.

8. Kawata T, Imanishi Y, Kobayashi K, Onoda N, Takemoto Y, Tahara $\mathrm{H}$, et al. Direct in vitro evidence of extracellular $\mathrm{Ca}^{2+}$ induced amino-terminal truncation of human parathyroid hormone (1-84) by human parathyroid cells. J Clin Endocrinol Metab. 2005;90:5774-8.

9. Gao P, Scheibel S, D'Amour P, John MR, Rao SD, SchmidtGayk H, Cantor TL. Development of a novel immunoradiometric assay exclusively for biologically active whole parathyroid hormone 1-84: implications for improvement of accurate assessment of parathyroid function. J Bone Miner Res. 2001;16:605-14.

10. Arakawa T, D'Amour P, Rousseau L, Brossard JH, Sakai M, Kasumoto $\mathrm{H}$, et al. Overproduction and secretion of a novel amino-terminal form of parathyroid hormone from a severe type of parathyroid hyperplasia in uremia. Clin J Am Soc Nephrol. 2006;1:525-31.

11. Kazama JJ, Omori T, Ei I, Ei K, Oda M, Maruyama H, et al. Circulating 1-84 PTH and large C-terminal PTH fragment levels in uremia. Clin Exp Nephrol. 2003;7:144-9.
12. Monier-Faugere MC, Geng Z, Mawad H, Friedler RM, Gao P, Cantor TL, Malluche HH. Improved assessment of bone turnover by the PTH-(1-84)/large C-PTH fragments ratio in ESRD patients. Kidney Int. 2001;60:1460-8.

13. Kazama JJ, Omori K, Higuchi N, Takahashi N, Ito Y, Maruyama $\mathrm{H}$, et al. Intact PTH assay overestimates true 1-84 PTH levels after maxacalcitol therapy in dialysis patients with secondary hyperparathyroidism. Nephrol Dial Transplant. 2004;19:892-7.

14. Fukagawa M, Yokoyama K, Koiwa F, Taniguchi M, Shoji T, Kazama JJ, et al. CKD-MBD Guideline Working Group: Japanese Society for Dialysis Therapy Clinical Practice Guideline for chronic kidney disease related mineral bone disease. Ther Apher Dial. 2013;17:247-88.

15. Valle C, Rodriguez M, Santamaría R, Almaden Y, Rodriguez $\mathrm{ME}$, Cañadillas $\mathrm{S}$, et al. Cinacalcet reduces the set point of the PTH-calcium curve. J Am Soc Nephrol. 2008;19:2430-6.

16. Martin KJ, Jüppner H, Sherrard DJ, Goodman WG, Kaplan MR, Nassar G, et al. First- and second-generation immunometric PTH assays during treatment of hyperparathyroidism with cinacalcet HCl. Kidney Int. 2005;68:1236-43.

17. de Francisco AL, Izquierdo M, Cunningham J, Piñera C, Palomar $\mathrm{R}$, Fresnedo GF, et al. Calcium-mediated parathyroid hormone release changes in patients treated with the calcimimetic agent cinacalcet. Nephrol Dial Transplant. 2008;23:2895-901.

18. GuidelineWorking Group. Japanese Society for Dialysis Therapy Clinical Practice Guideline for the management of secondary hyperparathyroidism in chronic dialysis patients. Ther Apher Dial. 2006;12:514-25.

19. Souberbielle JC, Boutten A, Carlier MC, Chevenne D, Coumaros G, Lawson-Body E, et al. Inter-method variability in PTH measurement: implication for the care of CKD patients. Kidney Int. 2006;70:345-50.

20. Kazama JJ, Yamamoto S, Kameda S, Maruyama H, Narita I, Shigematsu T, et al. Direct comparison between two 1-84PTH assays in dialysis patients. Nephron Clin Pract. 2005;99:c8-12. 